\title{
Analysis of the Influencing Factors of Energy Carbon Emission in Shaanxi Province Based on DEMATEL Method
}

\author{
Li Jinxing, Wang Jing*, Wang Meizhi \\ College of Economics and Management, Northwest A\&F University \\ Shanxi 712100, China

\section{基于 DEMATEL 方法的陕西省能源碳排放影响 因素分析}

\author{
李金星, 王静*, 王美知 \\ 西北农林科技大学经济管理学院 \\ 陕西 712100, 中国
}

\begin{abstract}
With the warming of the global climate and the increase of the greenhouse effect, it has seriously threatened the sustainable development of human beings. Many countries have adopted corresponding countermeasures to resolve this situation. China has also taken active measures and issued corresponding regulations and strategies to ease the status quo. Based on the basic data of Shaanxi Province from 2005 to 2016, an index system of four-dimensional and seven-variable was established, which includes resource endowments, technology levels, consumption patterns, and development stages. The DEMATEL method was used to analyze the influencing factors and effects of energy carbon emissions in Shaanxi Province. The results of the study indicate that resource endowments and population density are the most influential dimensions and indicators, respectively, and they are each a result and a causal factor. Finally, it put forward targeted policy recommendations to provide a reference for the low-carbon economy in Shaanxi Province.
\end{abstract}

Keywords—carbon emission; DEMATEL method; influence factor; Shaanxi Province

摘要一随着全球气候的变暖、温室效应的增加, 已经严重 威胁到了人类的可持续发展, 很多国家都采取了相应的对策 来解决这种状况, 我国也积极采取措施、出台相应的策略以 缓解现状。以陕西省 2005-2016 年相关基础数据建立资源禀赋、 技术水平、消费方式和发展阶段四维 17 个变量指标体系, 利 用 DEMATEL 方法对陕西省能源碳排放量影响因素和效应进 行分析。研究结果表明: 资源禀赋状况和人口密度分别是影 响度最强的维度和指标, 并且各为结果性影响因素和原因性 影响因素。最后据此提出了针对性的政策建议, 以期为陕西 省的低碳经济提供参考。

关键词—碳排放; DEMATEL 方法; 影响因素; 陕西省

\section{I. 引言}

随着新一轮工业革命兴起和经济的快速发展, 对能源 消耗的需求不断增加, 导致 CO2 排放过多而引起的温室
效应已经成为全世界关注的焦点问题。中国作为经济发 展的大国, 应对气候变化和控制温室气体排放成为当前 重要的任务。我国积极落实《联合国气候变化框架公约》 和《巴黎协定》, 根据经济发展状况, 国务院 2016 年 11 月印发了《“十三五”控制温室气体排放工作方案的通 知》, 以加快推进绿色低碳发展, 确保完成“十三五”规 划纲要确定的低碳发展目标任务, 推动二氧化碳排放 2030 年左右达到峰值并争取尽早达峰。

陕西省作为我国西部经济发展的大省和西部生态环境 建设的重点区域，积极响应“十三五”规划，于 2018 年 2 月 2 日印发《陕西省“十三五”控制温室气体排放工作实 施方案》，明确本省“十三五”控制温室气体排放的重点 工作和目标: 到 2020 年, 全省单位地区生产总值二氧化 碳排放比 2015 年下降 $18 \%$, 碳排放总量得到有效控制; 氢氟碳化物、甲烷等非二氧化碳温室气体控排力度进一 步加大[2]。陕西省作为低碳试点省份, 要完成减排目标, 就必须研究碳排放的影响因素, 才能有效的控制温室气 体的排放。

随着国家日益重视生态环境问题, 学者们也围绕生态 与经济发展问题开展了一系列研究。从世界角度看, 主 要探究世界主要国家碳排放影响因素[1]和测定主要国家 间[2]、相同发展水平国家间[3]和双边国家间 [4]的贸易 隐含碳排放的变动和影响因素。其次, 对于中国碳排放 的研究主要侧重于对国家整体化石能源碳排放影响因素 [5]、和驱动因素的研究、不同行业碳排放的测算及其影 响因素的探究[6]以及对省域碳排放强度[7]和空间效应[8] 的影响分析, 还有对家庭碳排放影响因素的研究[9]。但 是, 当前有关陕西碳排放的研究较少, 主要包括对经济 的低碳环保发展战略研究、碳排放及低碳化发展研究、 土地利用变化的碳排放效益研究、能源碳排放及预测研 究等。鉴于此, 本文运用 2005-2016 年《陕西省统计年

基金项目; 农户生产联结（PPL）机制及其关联性信用风险演化机理研究（71873101)，国家自然科学基金面上项目

作者简介: 李金星 (1991-), 女, 山西大同人, 硕士研究生, 研究专业: 农业经济管理, E-mai1: 826700956@qq. com;

王美知 (1992-), 女, 河南単城人, 硕士研究生, 研究专业: 农业经济管理, E-mai1: $\overline{2017051296 @ n w a f u}$. edu. cn。

*通讯作者:王 静 (1966-), 女, 陕西汉中人, 博士, 教授, 研究方向: 金融工程与农业投资, E-mai1: jing. wang@nwsuaf.edu. cn; 
鉴》和陕西省国民经济和社会发展统计公报，系统分析 陕西省碳排放影响因素。

建立有效的减排机制和实施针对的减排政策须明确以 下几个问题: 首先, 历史时期陕西省碳排放量的规律和 构成如何? 其次, 各个时期碳排放量由哪些影响和驱动 因素? 最后, 各个因素对碳排放量的影响程度和效应有 多大? 我们认为, 只有在回答了以上几个问题, 才能够 明确陕西省碳减排工作开展的重点领域, 进而推进减排 任务的高效完成。因此本文主要针对上述几个问题开展 讨论与分析, 从而为陕西省能源碳排放总量控制和强度 控制机制的构建提供理论依据和技术支撑。

\section{II. 研究方法及指标体系建立}

大多数研究采用指标分解的方法进行研究, 如 Kaya 恒等式的 LMDI 分解方法[10]、Divisia 指数分解法[11]、 Laspeyres 指数完全分解法[12]和结构分解法[13]等。对 碳排放空间差异及其影响因素研究的方法有 ESDAGWR 模型[14]、QAP 方法[15], 除此以外还有偏于质性 分析的主成分分析法、灰色关联性分析法以及 RMDEMATEL 方法。

DEMATEL(Decision Making Trial and Evaluation Laboratory), 决策试验与评价实验室分析法)是 1971 年 由美国 Bastille 实验室提出的一种分析系统中各因素关 系的一种建模方法, 该方法将图论和矩阵工具的组合应 用, 通过系统中因素之间的逻辑关系构建直接影响矩阵, 计算出每个因素的影响度和被影响度、中心度与原因度, 明确影响因素的地位角色及系统功能, 从而辨识整个系 统的关键因素。由于该方法是因素间相互关系评价及识 别的有效方法, 适合对复杂系统因素间的交互影响进行 研究，近些年在“三农问题、生态问题、企业管理以及社 会公共管理等多个研究领域得到广泛应用。方法上的改 进与创新层出不穷, 有模糊 DEMATEL 方法[16]、区间 数 DEMATEL 方法[17]、网络 DEMADEL 方法[18]以及 复合方法[19]。本文所运用的 DEMATEL 方法和步骤如 下:

步骤 1: 建立影响陕西省碳排放的指标体系, 如表 1 。

步骤 2: 考察不同影响因素间的影响关系, 及建立直 接影响矩阵 $\mathrm{A}$ 。

$$
A=\left[\begin{array}{lllc}
\mathrm{a}_{11} & \mathrm{a}_{12} & \cdots & \mathrm{a}_{1 j} \\
a_{21} & \mathrm{a}_{22} & \cdots & \mathrm{a}_{2 j} \\
\vdots & \vdots & \vdots & \vdots \\
a_{i 1} & \mathrm{a}_{i 2} & \cdots & a_{i j}
\end{array}\right]
$$

（1）中 $\alpha_{y}$ 表示两个因素之间的直接影响关系, 即影 响因素 $x i$ 对影响因素 $x j$ 的直接影响程度（i、 $j=1,2,3 \ldots \ldots 17)$ 。若 $i=j$, 则 $\alpha_{\mathrm{g}}=0$ 。

步骤 3: 计算规范化的直接影响矩阵 B。即:

$$
B=\min \left\{1 / \max _{i} \sum_{j=1}^{17} a_{i j}, 1 / \max _{j} \sum_{i=1}^{17} a_{i j}\right\} * A
$$

步骤 4: 计算各影响因素之间的总影响矩阵 C, (3) 式中, $C_{i j}$ 表示影响因素 $x i$ 对影响因素 $x j$ 的直接影响和 间接影响程度。

$$
C=B(I-B)^{-1}
$$

步骤 5: 计算各个影响因素的影响度和被影响度。将 总影响矩阵 $\mathrm{C}$ 的行和列的和分别表示为列向量 $\mathrm{r}$ 和 $\mathrm{S}$ 。

$$
\begin{aligned}
& r_{i}=\sum_{j=1}^{17} c_{i j} \\
& S_{j}=\sum_{i=1}^{17} c_{i j}
\end{aligned}
$$

（4）式中, $r_{i}$ 是影响因素 xi 的影响度;（5）式中, $s_{j}$ 是政策因素 $x i$ 的被影响度。 $r_{i}+s_{j}$ 代表影响因素 $x i$ 的由影 响度和被影响度相加得到的中心度, 表示该影响因素在 所有影响因素中所起作用的大小, 中心度越大, 表明该 影响因素陕西省碳排放总量的影响效应越大。 $\mathrm{r}_{\mathrm{i}-\mathrm{s}}$ 代表影 响因素 xi 的由影响度和被影响度相减得到的原因度。 $\mathrm{r}_{\mathrm{i}^{-}}$ $\mathrm{s}_{\mathrm{j}}>0$, 表明在在碳排放行为的影响效应中, 该影响因素 起主动作用, 称之为原因因素; $\mathrm{r}_{\mathrm{i}}-\mathrm{S}_{\mathrm{j}}<0$, 表明在在碳排 放行为的影响效应中, 该影响因素是被动的, 称之结果 因素。

步骤 6: 计算各一级指标的影响效应（影响度、被影 响度、中心度和原因度）。基于二级指标所属的不同的 一级指标, 将总影响矩阵 $\mathrm{C}$ 转化为 $\mathrm{C}_{\mathrm{X}}$ 矩阵。

$$
C_{X}=\left[\begin{array}{cccc}
C_{X}^{11} & \mathrm{C}_{X}^{12} & \mathrm{C}_{X}^{13} & \mathrm{C}_{X}^{14} \\
C_{X}^{21} & \mathrm{C}_{X}^{22} & \mathrm{C}_{X}^{23} & \mathrm{C}_{X}^{24} \\
C_{X}^{31} & \mathrm{C}_{X}^{32} & \mathrm{C}_{X}^{33} & \mathrm{C}_{X}^{34} \\
C_{X}^{41} & \mathrm{C}_{X}^{42} & \mathrm{C}_{X}^{43} & \mathrm{C}_{X}^{44}
\end{array}\right]
$$

(6) 式中

$$
C_{X}^{11}=\left[\begin{array}{llll}
C_{11} & \mathrm{C}_{12} & \mathrm{C}_{13} & \mathrm{C}_{14} \\
C_{12} & \mathrm{C}_{22} & \mathrm{C}_{23} & \mathrm{C}_{24} \\
C_{31} & \mathrm{C}_{32} & \mathrm{C}_{33} & \mathrm{C}_{34} \\
C_{41} & \mathrm{C}_{42} & \mathrm{C}_{43} & \mathrm{C}_{44}
\end{array}\right], C_{X}^{12}=\left[\begin{array}{llll}
C_{15} & \mathrm{C}_{16} & \mathrm{C}_{17} & \mathrm{C}_{18} \\
C_{25} & \mathrm{C}_{26} & \mathrm{C}_{27} & \mathrm{C}_{28} \\
\mathrm{C}_{35} & \mathrm{C}_{36} & \mathrm{C}_{37} & \mathrm{C}_{38} \\
C_{45} & \mathrm{C}_{46} & \mathrm{C}_{47} & \mathrm{C}_{48}
\end{array}\right]
$$

依次类推。

步骤 7: 将 $\mathrm{C}_{\mathrm{X}}$ 矩阵转化为标准化的 $\mathrm{C}_{\mathrm{X}}$ 矩阵。 $\mathrm{C}_{\mathrm{X}}$ 矩阵 的行和列的和分别表示四大一级指标的影响度和被影响 度。

$$
C_{X}^{a}=\left[\begin{array}{llll}
C_{X}^{a 11} & C_{X}^{a 12} & C_{X}^{a 13} & C_{X}^{a 14} \\
C_{X}^{a 21} & C_{X}^{a 22} & C_{X}^{a 23} & C_{X}^{a 24} \\
C_{X}^{a 31} & C_{X}^{a 32} & C_{X}^{a 33} & C_{X}^{a 34} \\
C_{X}^{a 41} & C_{X}^{a 42} & C_{X}^{a 43} & C_{X}^{a 44}
\end{array}\right]
$$

式中

$$
C_{X}^{a 11}=\frac{1}{16} \sum_{i=1}^{4} \sum_{j=1}^{4} C_{i j}, C_{X}^{a 12}=\frac{1}{16} \sum_{i=1}^{4} \sum_{j=5}^{8} C_{i j}
$$


依次类推。

\section{(2) 指标体系建立}

宋德勇[20]等利用时间序列数据对中国二氧化碳排放 影响因素时将因素分解为产出规模、能源结构、排放强 度、能源结构、产出结构、能源效率等因素。程叶青等 [21]运用空间计量的方法研究中国省域碳排放总量驱动 因素证明能源强度、能源结构、产业结构、城市化率对 中国能源消费碳排放具有重要影响。李新运等[6]在测算 中国行业碳排放量及其影响因素时, 建立了产业结构、 人口规模、能源强度、能源绩效、能源结构以及能源技 术进步等六维指标体系。

通过回顾文献, 国内学者对碳排放影响因素做了比较 全面、透彻的研究, 但是对作为西部生产和消费能源大 省的陕西省进行碳排放影响因素研究的文献相对较少, 而且将专门用于分析系统中各因素关系的 DEMATEL 方 法用于能源碳排放影响因素的文献也比较罕见。因此, 本文将采用 DEMATEL 方法对陕西省能源消费碳排放的 影响因素进行探究。

本文依据文献综述结果和指标设立的可获得性、全面 性、科学性和可比性原则, 建立两级指标体系, 一级指 标包含资源禀赋、技术水平、消费方式和发展阶段四个 维度, 二级指标是对一级指标的细化, 包含 17 个二级指 标，具体指标和计算方法如表 1 。

\section{III. 实证分析}

\section{(1) 数据来源和数据处理}

本文研究数据来源于 2005-2016 年《陕西省统计年鉴》 和陕西省国民经济和社会发展统计公报。数据进行了以 下三项处理。

处理一：指标单位碳排放产出的计算借鉴郝丽，姜创 业等的计算方法[22], 即:

能源消费碳排放 $=\sum$ (能源消费量 $\times$ 能源排放系数 $)$ 表 3 是不同影响因素对陕西省能源消费碳排放行为影 其中能源排放系数为各大能源碳排放系数标准的简单平
均数, 如表 2 。

处理二：直接影响矩阵的计算借鉴李中东、孙焕等的 研究成果，采用最小二乘法估计各政策因素间的直接影

表 2. 各种能源排放系数标准

\begin{tabular}{lllll|}
\hline \multicolumn{1}{c}{ 数据来源 } & 煤炭 & 石油 & 天然气 & 水电 \\
\hline DOE/EIA & 0.70 & 0.48 & 0.39 & 0.00 \\
日本能源经济研究所 & 0.76 & 0.48 & 0.45 & 0.00 \\
国家发改委能源所 & 0.75 & 0.59 & 0.44 & 0.00 \\
国家计委能源所 & 0.65 & 0.54 & 0.40 & 0.00 \\
国家科委气候变化项目 & 0.73 & 0.58 & 0.41 & 0.00 \\
国家环保局温室气体控制项目 0.75 & 0.58 & 0.44 & 0.00 \\
中国工程院 & 0.68 & 0.54 & 0.41 & 0.00 \\
平均值 & 0.72 & 0.56 & 0.42 & 0.00 \\
\hline
\end{tabular}

响系数, 而非通过传统的专家打分法。本文采用如下线 性回归模型:

$$
X_{j}=\beta_{0}+\beta_{i j} X_{j}+\varepsilon_{i j}
$$

(8) 式中, 回归系数 $\beta_{\mathrm{ij}}$ 表示影响因素 $\mathrm{x}_{\mathrm{i}}$ 重要程度分 值对因素 $\mathrm{x}_{\mathrm{j}}$ 重要程度分值的矢量影响, 即影响因素 $\mathrm{x}_{\mathrm{i}}$ 重 要程度的变化对因素 $\mathrm{x}_{\mathrm{j}}$ 重要程度所产生的影响; $\varepsilon_{\mathrm{ij}}$ 为误 差项。回归系数的 $\mathrm{t}$ 检验情况见附表 1, 在回归结果中, 回归系数 $\beta_{\mathrm{ij}}$ 的绝对值就是直接影响矩阵 $\mathrm{A}$ 中的 $\mathrm{a}_{\mathrm{ij}}$ 。附 表 1 显示的是不同影响因素之间的直接影响矩阵 $\mathrm{A}$ 。经 过 (2) 式、（3）式的计算, 可得出附表 2 所示的影响 能源碳排放因素之间的总影响矩阵 C。根据(4) （7) 式, 可以计算不同一级指标的影响效应（见表 3）。

处理三: 为了使防止各影响因素回归系数差异过大而 影响结果, 本研究通过改变单位, 使原始数值大小保证 在区间 $(0,1)$ 之间。

\section{(2) 研究结果分析}

响效应对的 DEMATEL 计算结果。从一级指标间的相互

表 1. 陕西省碳排放影响因素指标体系

\begin{tabular}{|c|c|c|c|}
\hline 一级指标 & & 二级指标 & 计算公式 \\
\hline \multirow{5}{*}{$\mathrm{X} 1$ 资源禀赋 } & $\mathrm{x} 1$ & 水电、风电等其他能发电占消费能源的比例 (\%) & （水电、风电等其他能发电/能源消费总量）*100\% \\
\hline & $\mathrm{x} 2$ & 人口密度 (千人/平方公里) & 常住人口数/总面积 \\
\hline & $\mathrm{x} 3$ & 有效灌溉面积占耕地面积的比重（\%） & （有效灌溉面积/耕地面积）*100\% \\
\hline & $\mathrm{x} 4$ & 森林覆盖率（\%) & -1 \\
\hline & $\times 5$ & 水资源总量 (千亿立方米) & - \\
\hline \multirow{4}{*}{$\mathrm{X} 2$ 技术水平 } & $\mathrm{x} 6$ & 单位碳排放的产出（十万元/吨碳） & （GDP 总量/能源消费碳排放量）* $100 \%$ \\
\hline & $\times 7$ & 单位能源的产出（十万元/吨标准煤） & 1/GDP 能耗 \\
\hline & $\mathrm{x} 8$ & 工业固体废物综合利用率（\%) & - \\
\hline & $\mathrm{x} 9$ & 城镇生活污水处理率（\%) & - \\
\hline \multirow{4}{*}{ X3 消费方式 } & $\mathrm{x} 10$ & 人均化石能源消费量（十吨标准煤/人） & 能源总消费量/常住人口数 \\
\hline & $\mathrm{x} 11$ & 教科文卫事业占财政支出的比例（\%) & $\begin{array}{c}\text { （科学、教育、文化和卫生支出总和/一般预算支 } \\
\text { 出）*100\% }\end{array}$ \\
\hline & $\mathrm{x} 12$ & 单位工业增加值能耗（十吨标准煤/万元） & - \\
\hline & $\mathrm{x} 13$ & 能源消费弹性系数 & - \\
\hline \multirow{4}{*}{ X4 发展阶段 } & $\mathrm{x} 14$ & 农村居民家庭恩格尔系数（\%） & - \\
\hline & $\mathrm{x} 15$ & GDP 增长率（\%） & - \\
\hline & $\mathrm{x} 16$ & 第三产业产出占总产出比重（\%) & - \\
\hline & $\mathrm{x} 17$ & 城市化率（\%) & (城镇人口/常住人口数)* $100 \%$ \\
\hline
\end{tabular}


影响看, 资源禀赋维度 (X1) 具有较高的影响度和被影 响度。这一结果表明, 资源禀赋状况对技术发展水平、 居民消费方式以及经济发展阶段的影响度最大，同时其 他也被其他因素影响最强。模型计算结果与陕西省经济 发展主要依赖资源能源开发和消费的现状相一致。

从一级指标对陕西省碳排放强度影响的重要性来看, 资源禀赋水平 $\mathrm{X} 1$ 的中心度为 0.0360 , 明显高于技术发 展水平、居民消费方式[23]和经济发展阶段的中心度。 这一结果表明, 陕西省资源禀赋状况对其能源碳排放强
度是 17 个指标中影响度最强的指标。即在资源禀赋维度 中, 人口数量通过影响其他资源禀赋指标来影响陕西省 能源碳排放的强度, 影响力最大; 在技术水平维度中, 单位碳排放产出效率对碳排放强度具有主动影响效应。 由此, 陕西省需要控制本省总人口数量, 协调人口、资 源与环境之间的矛盾; 提升城镇化水平，优化人口结构, 提高人口受教育水平; 倡导全民低碳生产生活理念, 大 力发展低碳农业, 提升全民低碳意识。积极推进技术进 步, 加快低碳技术研发与示范，加大低碳技术推广应用 力度。

表 3. DEMATEL 方法计算结果

\begin{tabular}{|c|c|c|c|c|c|c|c|c|c|}
\hline 一级指标 & $\mathrm{r}_{\mathrm{i}}$ & $\overline{S_{i}}$ & $\mathrm{r}_{\mathrm{i}}+\mathrm{s}_{\mathrm{i}}$ & $\mathrm{r}_{\mathrm{i}}-\mathrm{S}_{\mathrm{i}}$ & 二级指标 & $\mathrm{r}_{\mathrm{i}}$ & $\mathrm{S}_{\mathrm{i}}$ & $\mathrm{r}_{\mathrm{i}}+\mathrm{s}_{\mathrm{i}}$ & $\mathrm{r}_{\mathrm{i}}-\mathrm{S}_{\mathrm{i}}$ \\
\hline $\mathrm{X} 1$ & 0.0087 & 0.0273 & 0.0360 & -0.0187 & $\begin{array}{l}\mathrm{x} 1 \\
\mathrm{x} 2 \\
\mathrm{x} 3 \\
\mathrm{x} 4 \\
\mathrm{x} 5\end{array}$ & $\begin{array}{l}0.0102 \\
0.1564 \\
0.0060 \\
0.0011 \\
0.0000\end{array}$ & $\begin{array}{l}0.0001 \\
0.0000 \\
0.0000 \\
0.0016 \\
0.0003\end{array}$ & $\begin{array}{l}0.0103 \\
0.1564 \\
0.0060 \\
0.0026 \\
0.0003\end{array}$ & $\begin{array}{c}0.0101 \\
0.1564 \\
0.0059 \\
-0.0005 \\
-0.0003\end{array}$ \\
\hline $\mathrm{X} 2$ & 0.0001 & 0.0030 & 0.0032 & -0.0029 & $\begin{array}{l}\mathrm{x} 6 \\
\mathrm{x} 7 \\
\mathrm{x} 8 \\
\mathrm{x} 9\end{array}$ & $\begin{array}{l}0.0003 \\
0.0017 \\
0.0000 \\
0.0000\end{array}$ & $\begin{array}{l}0.0075 \\
0.0012 \\
0.0428 \\
0.1006\end{array}$ & $\begin{array}{l}0.0077 \\
0.0030 \\
0.0428 \\
0.1006\end{array}$ & $\begin{array}{c}-0.0072 \\
0.0005 \\
-0.0427 \\
-0.1006\end{array}$ \\
\hline $\mathrm{X} 3$ & 0.0001 & 0.0036 & 0.0037 & -0.0035 & $\begin{array}{l}\mathrm{x} 10 \\
\mathrm{x} 11 \\
\mathrm{x} 12 \\
\mathrm{x} 13\end{array}$ & $\begin{array}{l}0.0002 \\
0.0004 \\
0.0006 \\
0.0000\end{array}$ & $\begin{array}{l}0.0089 \\
0.0019 \\
0.0032 \\
0.0019\end{array}$ & $\begin{array}{l}0.0091 \\
0.0024 \\
0.0037 \\
0.0020\end{array}$ & $\begin{array}{l}-0.0087 \\
-0.0015 \\
-0.0026 \\
-0.0019\end{array}$ \\
\hline $\mathrm{X} 4$ & 0.0001 & 0.0002 & 0.0003 & -0.0001 & $\begin{array}{l}x 14 \\
x 15 \\
x 16 \\
x 17\end{array}$ & $\begin{array}{l}0.0003 \\
0.0006 \\
0.0003 \\
0.0002\end{array}$ & $\begin{array}{l}0.0061 \\
0.0020 \\
0.0002 \\
0.0002\end{array}$ & $\begin{array}{l}0.0064 \\
0.0026 \\
0.0005 \\
0.0005\end{array}$ & $\begin{array}{c}-0.0058 \\
-0.0013 \\
0.0000 \\
0.0000\end{array}$ \\
\hline
\end{tabular}

服最具有影响效用，在四大维度中处于核心地位。其次 是消费方式、技术水平和发展阶段。

从不同维度的之间的相互关系来看，其原因度均为负 值。说明这四方面对碳排放的影响并不是一个交叉复杂 的过程, 都属于结果性影响因素, 其被影响程度大于影 响程度。就 $\mathrm{X} 1 、 \mathrm{X} 2$ 的二级指标来看, $\mathrm{x} 1 \sim \mathrm{x} 5 、 \mathrm{x} 6 \sim \mathrm{x} 9$ 的原因度各有正有负, 说明资源禀赋和技术水平内部是 复杂的影响过程, 其中 $\mathrm{x} 1$ 人口密度和 $\mathrm{x} 7$ 单位能源的产 出分别在资源禀赋和技术水平维度中是重要的原因性指 标, 表明人口密度的大小和单位能源的产出效率在碳排 放过程中起到主动作用, 其他因素多起到被动影响的效 应。(如表 3 所示)

\section{IV. 结论与建议}

本文采用决策实验分析法（DEMATEL），基于 2005-2016 年陕西省的统计数据, 研究了资源禀赋状况、 技术发展水平、居民消费方式和经济发展阶段四维度对 陕西省能源碳排放的影响效应, 研究的主要结论及其建 议如下:

第一，在四个维度中，资源禀赋状况是影响陕西省碳 排放的最具影响效应的方面, 并是结果性影响因素, 即 控制资源能源的开发和使用是进行节能减排的最有效举 措，一方面加快传统“高耗能、高污染”产业结构调整和 技术升级，减少化石能源的消耗，提高能源的利用效率; 另一方面, 大力推进非化石能源（如，水电、风电、太 阳能和生物质能等）的开发，提高清洁能源消费比重。

第二, 人口密度和单位能源的产出分别在资源禀赋和 技术水平维度中是重要的原因性影响因素, 而且人口密

\section{致谢}

本研究受国家自然科学基金农户生产联结机制及其关 联性信用风险演化机理研究 (71873101) 资助。

$$
\text { 参考文献 }
$$

[1] 韩梦瑶, 刘卫东, 唐志鹏,等. 世界主要国家碳排放影响因素分析一 一基于变系数面板模型.资源科学, 2017,39(12):2420-2429.

[2] 蒋雪梅, 刘轶芳. 全球贸易隐含碳排放格局的变动及其影响因素. 统计研究，2013，30(9):29-36.

[3] 郑珍远, 李小敏, 张茂盛.中国与金砖国家贸易隐含碳比较研究. 亚太经济，2018(2).

[4] 赵玉焕, 王淞. 基于技术异质性的中日贸易隐含碳测算及分析. 北 京理工大学学报:社会科学版, 2014, 16(1):12-18.

[5] 孙叶飞, 周敏.中国能源消费碳排放与经济增长脱钩关系及驱动因 素研究.经济与管理评论,2017(6):21-30.

[6] 李新运,吴学锰,马俏俏.我国行业碳排放量测算及影响因素的结构 分解分析.统计研究, 2014,31(1):56-62.

[7] 吴玉鸣, 吕佩蕾.空间效应视角下中国省域碳排放总量的驱动因素 分析. 桂海论从, 2013(1):40-45.

[8] 邹秀萍, 陈劭锋, 宁氷, 等. 中国省级区域碳排放影响因素的实证分 析. 生态经济(中文版), 2009(3):34-37.

[9] 叶红, 潘玲阳, 陈峰,等. 城市家庭能耗直接碳排放影响因素—— 厦门岛区为例. 生态学报, 2010, 30(14):3802-3811.

[10] 梁大鹏, 刘天森, 李一军. 基于 LMDI 模型的金砖五国二氧化碳排 放成本及其影响因素比较研究. 资源科学, 2015, 37(12):2319-2329.

[11] 王圣, 王慧敏, 陈辉, 等. 基于 Divisia 分解法的江苏沿海地区碳排放 影响因素研究. 长江流域资源与环境, 2011, 20(10):1243-1247.

[12] 路正南, 杨洋, 王健. 基于 Laspeyres 分解法的中国碳生产率影响因 素解析. 工业技术经济, 2014(8):82-90.

[13] 朱勤, 彭希哲, 吴开亚. 基于结构分解的居民消费品载能碳排放变 动分析. 数量经济技术经济研究, 2012(1):65-77.

[14] 胡艳兴, 潘竟虎, 王怡睿. 基于 ESDA-GWR 的 1997-2012 年中国 省域能源消费碳排放时空演变特征. 环境科学学报, 2015, 35(6):1896-1906 
[15] 杨桂元, 吴齐, 涂洋. 中国省际碳排放的空间关联及其影响因素研 究——基于社会网络分析方法. 商业经济与管理, 2016(4):56-68.

[16] 金卫健, 胡汉辉. 模糊 DEMATEL 方法的拓展应用. 统计与决策, 2011，(23):170-171

[17] 高沛然, 卢新元.基于区间数的拓展 DEMATEL 方法及其应用研 究.运筹与管理, 2014, (01):44-50.

[18] 方爱丽.基于复杂网络理论的投入产出关联分析.青岛大学, 2008 .

[19] 唐丽艳, 周建林, 王国红.基于 DEMATEL-ANP 的产学研集成创 新评价研究一一大连双 D 港企业为例.科学学与科学技术管理, 2013, (12):87-96.

[20] 宋德勇, 卢忠宝. 中国碳排放影响因素分解及其周期性波动研究. 中国人口・资源与环境, 2009, 19(3):18-24.
[21] 程叶青, 王哲野, 张守志, 等. 中国能源消费碳排放强度及其影响因 素的空间计量. 地理学报, 2013, 68(10):1418-1431.

[22] 郝丽, 姜创业, 孙娴, 等.陕西省能源消费碳排放影响因素分析 与政策启示.水土保持研究，2013，20(6):326-332.

[23] Gary Webb, Alex Hendry, Bruce Armstrong, Robyn McDermott, Boyd Swinburn, Egger GarryExploring the Effects of Personal Carbon Trading (PCT) System on Carbon Emission and Health Issues: A Preliminary Study on the Norfolk Island,2014,4(1):1-11. 
附表 1 各影响因素之间的直接影响矩阵 (A)

\begin{tabular}{|c|c|c|c|c|c|c|c|c|c|c|c|c|c|c|c|c|c|}
\hline & $\mathrm{x} 1$ & $x 2$ & $\mathrm{x} 3$ & $\mathrm{x} 4$ & $\mathrm{x} 5$ & $\mathrm{x} 6$ & $\mathrm{x} 7$ & $\mathrm{x} 8$ & $\mathrm{x} 9$ & $\mathrm{x} 10$ & $\mathrm{x} 11$ & $\mathrm{x} 12$ & $\mathrm{x} 13$ & $\mathrm{x} 14$ & $\mathrm{x} 15$ & $\mathrm{x} 16$ & $\mathrm{x} 17$ \\
\hline $\mathrm{x} 1$ & 0.000 & $0.249 * * *$ & $0.605^{*}$ & $3.054 * * *$ & 0.821 & $5.995^{* *}$ & $2.500 * * *$ & $14.283^{* *}$ & $23.018^{* *}$ & $6.642 * *$ & 2.430 & $3.766 * *$ & 2.411 & $5.032 *$ & $3.708^{* * *}$ & 1.541 & 1.541 \\
\hline $\mathrm{x} 2$ & $2.814 * * *$ & 0.000 & 1.716 & $10.962 *$ & 0.006 & $24.040^{* * *}$ & $9.748^{* * *}$ & $58.189^{* * *}$ & $87.926^{* * *}$ & $26.260^{* * *}$ & $12.531^{* *}$ & $15.710^{* * *}$ & 9.487 & $21.984 * * *$ & $12.221^{* * *}$ & 3.931 & 3.931 \\
\hline $\mathrm{x} 3$ & $0.714 *$ & 0.179 & 0.000 & $2.367 *$ & 3.402 & $5.002 *$ & $1.919^{*}$ & 8.477 & 17.756 & $5.340^{*}$ & 2.380 & 3.058 & 5.498 & 3.312 & 2.350 & 0.344 & 0.344 \\
\hline $\mathrm{x} 4$ & $0.223 * * *$ & $0.071^{* * *}$ & $0.146^{*}$ & 0.000 & 0.996 & $1.969^{* * *}$ & $0.772 * * *$ & $4.417^{* * *}$ & $7.575^{* * *}$ & $2.000^{* * *}$ & $0.799 *$ & $1.263^{* * *}$ & 1.615 & $1.732^{* * *}$ & $0.868^{* *}$ & 0.042 & 0.042 \\
\hline $\mathrm{x} 5$ & 0.004 & 0.002 & 0.130 & 0.062 & 0.000 & 0.115 & 0.035 & 0.276 & 0.513 & 0.059 & 0.514 & 0.105 & $0.836^{*}$ & 0.149 & 0.073 & $0.134 *$ & $0.134^{*}$ \\
\hline $\mathrm{x} 6$ & $0.100 * *$ & $0.035^{* * *}$ & $0.071 *$ & $0.050^{* * *}$ & 0.425 & 0.000 & $0.381 * * *$ & $2.226^{* * *}$ & $3.728^{* * *}$ & $1.024 * * *$ & $0.051^{* *}$ & $0.648^{* * *}$ & 0.976 & $0.878^{* * *}$ & $0.414^{* *}$ & 0.000 & 0.000 \\
\hline $\mathrm{x} 7$ & $0.278 * * *$ & $0.096^{* * *}$ & $0.181 *$ & $1.175 * * *$ & 0.861 & $2.543 * * *$ & 0.000 & $5.868 * * *$ & $9.444 * * *$ & $2.671^{* * * *}$ & $0.126^{* *}$ & $1.671^{* * *}$ & 2.115 & $2.274 * * *$ & $1.137 * * *$ & 0.156 & 0.156 \\
\hline $\mathrm{x} 8$ & $0.043 * *$ & $0.016^{* * * *}$ & 0.022 & $0.184 * * *$ & 0.186 & $0.406^{* *}$ & $0.160^{* * *}$ & 0.000 & $1.522^{* * *}$ & $0.425 * * *$ & $0.204 * *$ & $0.267^{* * * *}$ & 0.308 & $0.370^{* * *}$ & $0.184^{* *}$ & 0.031 & 0.031 \\
\hline $\mathrm{x} 9$ & $0.027^{* *}$ & $0.009^{* * * *}$ & 0.017 & $0.120 * * *$ & 0.132 & $0.259 * * *$ & $0.098^{* * * *}$ & $0.581 * * *$ & 0.000 & $0.263 * * *$ & $0.129 * *$ & $0.165^{* * *}$ & 0.220 & $0.229 * * *$ & $0.109 * *$ & 0.003 & 0.003 \\
\hline $\mathrm{x} 10$ & $0.101 * *$ & $0.035^{* * *}$ & $0.069^{*}$ & $0.418 * * *$ & 0.199 & $0.940^{* * *}$ & $0.367 * * *$ & $2.140^{* * *}$ & $3.462 * * *$ & 0.000 & $0.497 * * *$ & $0.617 * * *$ & 0.708 & $0.840 * * *$ & $0.429 * * *$ & 0.066 & 0.066 \\
\hline $\mathrm{x} 11$ & 0.103 & $0.047^{* *}$ & 0.086 & $0.464 *$ & 0.084 & $1.311^{* *}$ & $0.480^{* *}$ & $2.857^{* *}$ & $4.717^{* *}$ & $1.381^{* * *}$ & 0.000 & $0.851^{* *}$ & 1.490 & $1.188^{* *}$ & 0.490 & 0.016 & 0.016 \\
\hline $\mathrm{x} 12$ & $0.142 * *$ & $0.052 * * *$ & 0.098 & $0.652 * * *$ & 0.879 & $1.470^{* * * *}$ & $0.568^{* * *}$ & $3.322 * * *$ & $5.372 * * *$ & $1.525^{* * *}$ & $0.757^{* *}$ & 0.000 & 1.850 & $1.326^{* * *}$ & $0.564 *$ & 0.008 & 0.008 \\
\hline $\mathrm{x} 13$ & 0.005 & 0.002 & 0.010 & 0.049 & $0.409 *$ & 0.129 & 0.042 & 0.224 & 0.420 & 0.102 & 0.077 & 0.108 & 0.000 & 0.095 & 0.032 & 0.083 & 0.083 \\
\hline $\mathrm{x} 14$ & $0.099 *$ & $0.038^{* * *}$ & 0.055 & $0.468^{* * *}$ & 0.650 & $1.042^{* * * *}$ & $0.404 * * *$ & $2.408 * * *$ & $3.905^{* * *}$ & $1.086^{* * *}$ & $0.553 * *$ & $0.694 * * *$ & 0.850 & 0.000 & 0.421 & 0.020 & 0.020 \\
\hline $\mathrm{x} 15$ & $0.217 * * *$ & $0.063^{* * *}$ & 0.116 & $0.696^{* *}$ & 0.947 & $1.456^{* *}$ & $0.599^{* * *}$ & $3.554^{* *}$ & $5.515^{* *}$ & $1.643^{* * *}$ & 0.676 & $0.875^{*}$ & 0.838 & $1.249^{* *}$ & 0.000 & 0.434 & 0.434 \\
\hline $\mathrm{x} 16$ & 0.143 & 0.323 & 0.027 & 0.054 & $2.766^{*}$ & 0.001 & 0.130 & 0.934 & 0.248 & 0.398 & 0.034 & 0.020 & 3.498 & 0.095 & 0.688 & 0.000 & $1.000^{* * *}$ \\
\hline $\mathrm{x} 17$ & 0.138 & 0.329 & 0.063 & 0.056 & $1.988^{*}$ & 0.009 & 0.131 & 1.998 & 0.268 & 0.398 & 0.036 & 0.020 & 3.511 & 0.078 & 0.068 & $1.000^{* * *}$ & $0.000^{* *}$ \\
\hline
\end{tabular}

附表 2 影响陕西省能源碳排放因素的综合影响矩阵 (C)

\begin{tabular}{|c|c|c|c|c|c|c|c|c|c|c|c|c|c|c|c|c|c|}
\hline & $\mathrm{x} 1$ & $\mathrm{x} 2$ & $\mathrm{x} 3$ & $\mathrm{x} 4$ & $\mathrm{x} 5$ & $x 6$ & $x 7$ & $\mathrm{x} 8$ & $\mathrm{x} 9$ & $\mathrm{x} 10$ & $\mathrm{x} 11$ & $\mathrm{x} 12$ & $\mathrm{x} 13$ & $\mathrm{x} 14$ & $\mathrm{x} 15$ & x16 & $\mathrm{x} 17$ \\
\hline $\mathrm{x} 1$ & $0.00 \mathrm{E}+00$ & 7.12E-07 & 4.13E-06 & $1.05 \mathrm{E}-04$ & $8.51 \mathrm{E}-06$ & 4.09E-04 & $7.10 \mathrm{E}-05$ & $2.32 \mathrm{E}-03$ & $6.02 \mathrm{E}-03$ & $5.01 \mathrm{E}-04$ & $6.74 \mathrm{E}-05$ & $1.62 \mathrm{E}-04$ & $6.99 \mathrm{E}-05$ & $2.89 \mathrm{E}-04$ & $1.55 \mathrm{E}-04$ & $2.65 \mathrm{E}-05$ & $2.65 \mathrm{E}-05$ \\
\hline $\mathrm{x} 2$ & $8.99 \mathrm{E}-05$ & $0.00 \mathrm{E}+00$ & $3.35 \mathrm{E}-05$ & $1.36 \mathrm{E}-03$ & 3.12E-08 & $6.56 \mathrm{E}-03$ & $1.08 \mathrm{E}-03$ & $3.84 \mathrm{E}-02$ & 8.79E-02 & 7.82E- 03 & $1.77 \mathrm{E}-03$ & $2.80 \mathrm{E}-03$ & $1.08 \mathrm{E}-03$ & $5.48 \mathrm{E}-03$ & $1.69 \mathrm{E}-03$ & 1.73E-04 & $1.73 \mathrm{E}-04$ \\
\hline $\mathrm{x} 3$ & $5.75 \mathrm{E}-06$ & $3.67 \mathrm{E}-07$ & $0.00 \mathrm{E}+00$ & $6.34 \mathrm{E}-05$ & $1.30 \mathrm{E}-04$ & $2.84 \mathrm{E}-04$ & 4.19E- 05 & $8.26 \mathrm{E}-04$ & $3.59 \mathrm{E}-03$ & $3.24 \mathrm{E}-04$ & $6.42 \mathrm{E}-05$ & $1.06 \mathrm{E}-04$ & $3.42 \mathrm{E}-04$ & $1.26 \mathrm{E}-04$ & $6.27 \mathrm{E}-05$ & $1.37 \mathrm{E}-06$ & $1.37 \mathrm{E}-06$ \\
\hline $\mathrm{x} 4$ & $5.63 \mathrm{E}-07$ & $5.70 \mathrm{E}-08$ & 2.44E-07 & $0.00 \mathrm{E}+00$ & $1.12 \mathrm{E}-05$ & 4.40E-05 & $6.76 \mathrm{E}-06$ & $2.21 \mathrm{E}-04$ & $6.50 \mathrm{E}-04$ & 4.54E-05 & 7.27E-06 & $1.81 \mathrm{E}-05$ & $2.97 \mathrm{E}-05$ & $3.40 \mathrm{E}-05$ & $8.57 \mathrm{E}-06$ & $2.24 \mathrm{E}-08$ & $2.24 \mathrm{E}-08$ \\
\hline $\mathrm{x} 5$ & $1.93 \mathrm{E}-10$ & $3.49 \mathrm{E}-11$ & $1.86 \mathrm{E}-07$ & 4.40E- 08 & $0.00 \mathrm{E}+00$ & $1.57 \mathrm{E}-07$ & $1.47 \mathrm{E}-08$ & $8.96 \mathrm{E}-07$ & $3.07 \mathrm{E}-06$ & 4.40E-08 & 2.92E-06 & $1.28 \mathrm{E}-07$ & $7.80 \mathrm{E}-06$ & $2.54 \mathrm{E}-07$ & $6.17 \mathrm{E}-08$ & 2.01E-07 & $2.01 \mathrm{E}-07$ \\
\hline $\mathrm{x} 6$ & $1.13 \mathrm{E}-07$ & $1.41 \mathrm{E}-08$ & 5.63E- 08 & 3.44E-08 & $2.05 \mathrm{E}-06$ & $0.00 \mathrm{E}+00$ & $1.64 \mathrm{E}-06$ & $5.60 \mathrm{E}-05$ & $1.57 \mathrm{E}-04$ & 1.19E-05 & $3.67 \mathrm{E}-08$ & $4.75 \mathrm{E}-06$ & $1.07 \mathrm{E}-05$ & 8.70E-06 & $1.94 \mathrm{E}-06$ & $9.01 \mathrm{E}-12$ & $9.01 \mathrm{E}-12$ \\
\hline $\mathrm{x} 7$ & 8.73E-07 & $1.04 \mathrm{E}-07$ & $3.71 \mathrm{E}-07$ & $1.56 \mathrm{E}-05$ & 8.54E-06 & 7.32E-05 & $0.00 \mathrm{E}+00$ & $3.90 \mathrm{E}-04$ & $1.01 \mathrm{E}-03$ & $8.08 \mathrm{E}-05$ & $2.25 \mathrm{E}-07$ & $3.16 \mathrm{E}-05$ & $5.09 \mathrm{E}-05$ & $5.85 \mathrm{E}-05$ & $1.47 \mathrm{E}-05$ & 2.82E-07 & $2.82 \mathrm{E}-07$ \\
\hline $\mathrm{x} 8$ & 2.13E-08 & 2.77E-09 & $5.48 \mathrm{E}-09$ & $3.81 \mathrm{E}-07$ & 3.93E-07 & $1.87 \mathrm{E}-06$ & $2.91 \mathrm{E}-07$ & $0.00 \mathrm{E}+00$ & $2.63 \mathrm{E}-05$ & $2.05 \mathrm{E}-06$ & $4.71 \mathrm{E}-07$ & $8.10 \mathrm{E}-07$ & $1.09 \mathrm{E}-06$ & $1.55 \mathrm{E}-06$ & $3.86 \mathrm{E}-07$ & $1.07 \mathrm{E}-08$ & $1.07 \mathrm{E}-08$ \\
\hline $\mathrm{x} 9$ & 8.06E-09 & $9.22 \mathrm{E}-10$ & 3.46E-09 & $1.63 \mathrm{E}-07$ & $1.96 \mathrm{E}-07$ & 7.62E-07 & $1.10 \mathrm{E}-07$ & 3.82E-06 & $0.00 \mathrm{E}+00$ & 7.83E-07 & $1.87 \mathrm{E}-07$ & $3.08 \mathrm{E}-07$ & $5.52 \mathrm{E}-07$ & $5.95 \mathrm{E}-07$ & $1.35 \mathrm{E}-07$ & 1.33E-10 & $1.33 \mathrm{E}-10$ \\
\hline 10 & $1.17 \mathrm{E}-07$ & $1.43 \mathrm{E}-08$ & 5.42E- 08 & $1.98 \mathrm{E}-06$ & 4.70E-07 & $1.00 \mathrm{E}-05$ & $1.53 \mathrm{E}-06$ & $.20 \mathrm{E}-05$ & $1.36 \mathrm{E}-04$ & $0.00 \mathrm{E}+00$ & $2.78 \mathrm{E}-06$ & 4.32E-06 & $5.75 \mathrm{E}-06$ & 8.00E-06 & $2.09 \mathrm{E}-06$ & 4.94E-08 & 4.94E-08 \\
\hline x11 & $1.22 \mathrm{E}-07$ & $2.51 \mathrm{E}-08$ & 8.34E-08 & $2.46 \mathrm{E}-06$ & $9.61 \mathrm{E}-08$ & $1.95 \mathrm{E}-05$ & $2.61 \mathrm{E}-06$ & $9.27 \mathrm{E}-05$ & $2.52 \mathrm{E}-04$ & $2.16 \mathrm{E}-05$ & $0.00 \mathrm{E}+00$ & $8.21 \mathrm{E}-06$ & $2.50 \mathrm{E}-05$ & $1.60 \mathrm{E}-05$ & $2.75 \mathrm{E}-06$ & $3.36 \mathrm{E}-09$ & 3.36E-09 \\
\hline$x 12$ & $2.30 \mathrm{E}-07$ & $3.12 \mathrm{E}-08$ & $1.09 \mathrm{E}-07$ & $4.82 \mathrm{E}-06$ & $8.70 \mathrm{E}-06$ & $2.45 \mathrm{E}-05$ & $3.66 \mathrm{E}-06$ & $1.25 \mathrm{E}-04$ & $3.28 \mathrm{E}-04$ & 2.64E-05 & $6.46 \mathrm{E}-06$ & $0.00 \mathrm{E}+00$ & $3.85 \mathrm{E}-05$ & $1.99 \mathrm{E}-05$ & $3.63 \mathrm{E}-06$ & 1.13E-09 & 1.13E-09 \\
\hline$x 13$ & $3.40 \mathrm{E}-10$ & $4.43 \mathrm{E}-11$ & $1.22 \mathrm{E}-09$ & $2.71 \mathrm{E}-08$ & $1.86 \mathrm{E}-06$ & $1.90 \mathrm{E}-07$ & 2.01E-08 & $5.76 \mathrm{E}-07$ & $2.01 \mathrm{E}-06$ & $1.20 \mathrm{E}-07$ & $6.79 \mathrm{E}-08$ & $1.32 \mathrm{E}-07$ & $0.00 \mathrm{E}+00$ & $1.03 \mathrm{E}-07$ & $1.17 \mathrm{E}-08$ & $7.68 \mathrm{E}-08$ & 7.68E-08 \\
\hline $\mathrm{x} 14$ & $1.12 \mathrm{E}-07$ & 1.67E-08 & $3.54 \mathrm{E}-08$ & $2.48 \mathrm{E}-06$ & 4.75E-06 & $1.23 \mathrm{E}-05$ & $1.85 \mathrm{E}-06$ & $6.57 \mathrm{E}-05$ & $1.73 \mathrm{E}-04$ & $1.34 \mathrm{E}-05$ & 3.44E-06 & $5.45 \mathrm{E}-06$ & $8.24 \mathrm{E}-06$ & $0.00 \mathrm{E}+00$ & $2.02 \mathrm{E}-06$ & $5.20 \mathrm{E}-09$ & $5.20 \mathrm{E}-09$ \\
\hline $\mathrm{x} 15$ & $5.31 \mathrm{E}-07$ & $4.59 \mathrm{E}-08$ & $1.54 \mathrm{E}-07$ & $5.48 \mathrm{E}-06$ & $1.02 \mathrm{E}-05$ & $2.41 \mathrm{E}-05$ & 4.07E-06 & $1.44 \mathrm{E}-04$ & $3.46 \mathrm{E}-04$ & 3.06E-05 & $5.19 \mathrm{E}-06$ & $8.74 \mathrm{E}-06$ & $8.25 \mathrm{E}-06$ & $1.78 \mathrm{E}-05$ & $0.00 \mathrm{E}+00$ & $2.10 \mathrm{E}-06$ & $2.10 \mathrm{E}-06$ \\
\hline x16 & $2.32 \mathrm{E}-07$ & $1.15 \mathrm{E}-06$ & 9.34E-09 & 4.26E-08 & $8.47 \mathrm{E}-05$ & $6.62 \mathrm{E}-10$ & $2.11 \mathrm{E}-07$ & $1.06 \mathrm{E}-05$ & $1.09 \mathrm{E}-06$ & $1.94 \mathrm{E}-06$ & $2.23 \mathrm{E}-08$ & $1.03 \mathrm{E}-08$ & $1.36 \mathrm{E}-04$ & $1.38 \mathrm{E}-07$ & $5.35 \mathrm{E}-06$ & $0.00 \mathrm{E}+00$ & $1.11 \mathrm{E}-05$ \\
\hline x17 & $2.16 \mathrm{E}-07$ & $1.20 \mathrm{E}-06$ & $4.59 \mathrm{E}-08$ & 4.65E- 08 & 4.39E-05 & 4.70E-09 & 2.13E-07 & 4.59E-05 & $1.23 \mathrm{E}-06$ & 1.93E-06 & 2.32E-08 & $1.02 \mathrm{E}-08$ & $1.37 \mathrm{E}-04$ & $9.84 \mathrm{E}-08$ & $6.70 \mathrm{E}-08$ & 1.11E-05 & $0.00 \mathrm{E}+00$ \\
\hline
\end{tabular}

